Pacific Journal of Mathematics

CLASSIFICATION OF ALGEBRAIC SURFACES WITH
SECTIONAL GENUS LESS THAN OR EQUAL TO SIX

RATIONAL SUMAC 


\title{
CLASSIFICATION OF ALGEBRAIC SURFACES WITH SECTIONAL GENUS LESS THAN OR EQUAL TO SIX. I: RATIONAL SURFACES
}

\section{ELVIRA LAURA LIVORNI}

\begin{abstract}
Using Sommese's results on the adjunction process we give a biholomorphic classification of rational algebraic surfaces with the genus of a hyperplane section less than or equal to six.
\end{abstract}

Introduction. In this paper we have given a biholomorphic classification of smooth, connected, projective, rational surfaces $X$ with smooth hyperplane section $C$ such that the genus $g$ of $C$ is less than or equal to six. In 1936, L. Roth in [R] had given a birational classification of such algebraic surfaces with $g$ less than or equal to six. Our biholomorphic classification goes considerably beyond his classification. Let $L=[C]$ for some hyperplane section $C$. Since in the cases in which $g=0,1, X$ has been completely classified by Del Pezzo [N], we need only to consider the cases in which $g=2, \ldots, 6$. Since the cases $g=2,3,4$ follow very easily from A. J. Sommese [So], the really interesting cases are $g=5$, 6. Our classification has a slight overlap with P. Ionescu [Io]. Our classification is essentially based on the adjunction process which was introduced by the Italian school and which has been particularly studied by Sommese [So]. Our notations are as in [So] except for the following. $X$ will denote a smooth, connected, projective, rational surface and $L$ a very ample line bundle on $X$. Let $\bar{L}=K_{X} \otimes L$. Then $\phi_{\bar{L}}$ is the map given by the sections of the line bundle $\bar{L}$. Sommese, [So, (2.0.1) pg 390], has proved that $\operatorname{dim} \phi_{\bar{L}}(X)=0$ if and only if $g=1$. If $X$ is rational then $\operatorname{dim} \phi_{\bar{L}}(X)=-\infty$ if and only if $h^{1,0}(X)=\operatorname{dim} H^{1}\left(X, \vartheta_{X}\right)=0$ and in this case $(X, L)$ has been classified by Del Pezzo, (0.6). Let $\phi_{\bar{L}}=r \circ s$ be the Remmert-Stein factorization of $\phi_{\bar{L}}$. In the case in which $\operatorname{dim} \phi_{\bar{L}}(X)=1$, it follows from Sommese [So, (2.1.1) pg 390] that, since $h^{1,0}(X)=0$ in our case, $s$ is an embedding. If $\operatorname{dim} \phi_{L}(X)=2$, Sommese [So, (2.3) pg 392], has proved that there exists a pair $(\hat{X}, \hat{L})$ such that:

(a) $X$ is obtained by blowing up a finite set of points $F$ of $\hat{X}, \pi$ : $X \rightarrow \hat{X}$.

(b) Every smooth hyperplane section $C \in|L|$ is the proper transform of a smooth hyperplane section $\hat{C} \in|\hat{L}-F|$. 
(c) $\hat{L}$ is ample and is very ample if $h^{1}(L)=0$.

(d) $K_{\hat{X}} \otimes \hat{L}$ is, in our case, very ample except in the two cases (2.5.1) and (2.5.2) of [So, pg 394].

Let $L^{\prime}=K_{\hat{X}} \otimes \hat{L}$ and $\phi_{L^{\prime}}=\phi_{K_{\hat{X}} \otimes \hat{L}}$. Then $\phi_{\bar{L}}=\phi_{L^{\prime}} \circ \pi$.

We call $\hat{X}$ the minimal model of $X$ relative to $L$. It has the property that there is no irreducible curve $\mathscr{P} \subset \hat{X}$ such that $\mathscr{P} \cdot \mathscr{P}=-1$ and $\hat{L} \cdot \mathscr{P}=1$. We call $(\hat{X}, \hat{L})$ the minimal pair of $(X, L)$. Note that since $L \cdot \mathscr{P}=1, \hat{C}$ is smooth. Our main goal is to classify the pairs $(\hat{X}, \hat{L})$ and eventually the pairs $(X, L)$. What makes the rational case interesting is that, by [So, (3.1) pg 395], $\phi_{L^{\prime}}$ is an embedding except for special cases that can be classified. This allows us to use a recursive method to classify the pairs $(\hat{X}, \hat{L})$. We would like to give an example of how the method works. To do this we fix the following notation. Let $d=L \cdot L, g=g(L)$, $\hat{d}=\hat{L} \cdot \hat{L}, d^{\prime}=L^{\prime} \cdot L^{\prime}$ and $g^{\prime}=g\left(L^{\prime}\right)$. Suppose that we are considering the case $\operatorname{dim} \phi_{L}^{-}(X)=2, g=6$ and that we have obtained the invariants $\hat{d}=10, g^{\prime}=4, d^{\prime}=8, \hat{c}_{1}^{2}=-3$. Then we look at the classification for $g=4$. Since there is no surface with sectional genus four and such that $d-c_{1}^{2}=d^{\prime}-\hat{c}_{1}^{2}=11$, we conclude that such a surface doesn't exist. Unfortunately, it starts to happen when $g=6$ that, as the genus $g$ grows, $g^{\prime}$ becomes greater than or equal to $g$ when we iterate the adjunction process. This is essentially the reason which makes it very difficult to use this method to try to extend the classification beyond $g=6$. We summarize in the following tables the results which we have obtained. When $\operatorname{dim} \phi_{L}(X)=1,(\hat{X}, \hat{L})$ denotes a minimal model and its relative line bundle. We thank Andrew J. Sommese for suggesting the problem and for his valuable advice.

0. Background material. Since most of our background material is as in [So], we will collect only material which cannot be found in [So]. We first state the following proposition which can be found in [R].

(0.1) Proposition. Let $L$ be a line bundle on a smooth, connected, projective surface $X$. Then:

(1) $d^{\prime}=g^{\prime}+g-2$,

(2) $d d^{\prime} \leq 4(g-1)^{2}$,

(3) $d+d^{\prime}=c_{1}^{2}+4(g-1)$.

Proof. To prove (1) we use the adjunction formula for the line bundles $L$ and $K_{X} \otimes L$ and the fact that

$$
d^{\prime}=\left(K_{X}+L\right) \cdot\left(K_{X}+L\right)=c_{1}^{2}-d+4 g-4 .
$$




\begin{tabular}{|c|c|c|c|c|c|c|c|c|c|c|}
\hline $\begin{array}{c}0- \\
1 \\
1 \\
0 \\
0 \\
0 \\
0 \\
0\end{array}$ & $\begin{array}{l}\infty \\
1\end{array}$ & $n$ & & 0 & 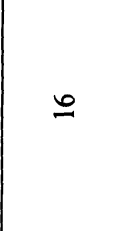 & $\forall$ & $\infty$ & $\simeq$ & $\underline{0}$ & ్ㅗ \\
\hline 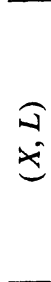 & $\begin{array}{c}x \\
11 \\
x\end{array}$ & $\begin{array}{l}x \\
11 \\
x\end{array}$ & $\underset{x}{11}$ & 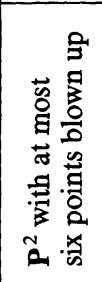 & 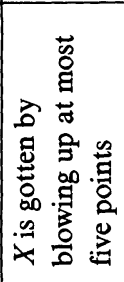 & 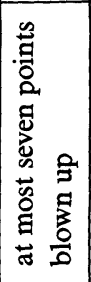 & 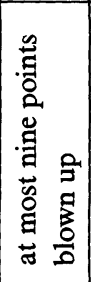 & 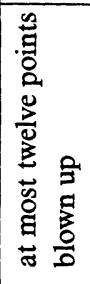 & 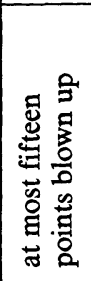 & 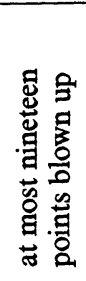 \\
\hline$\frac{1}{x}$ & 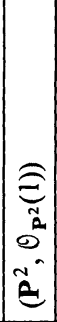 & $\begin{array}{c}\widehat{\widehat{d}} \\
\tilde{a} \\
\tilde{\sigma} \\
\tilde{\tilde{s}} \\
\tilde{\omega}\end{array}$ & 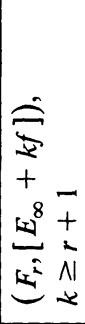 & 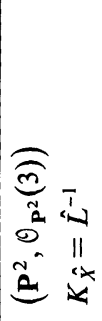 & 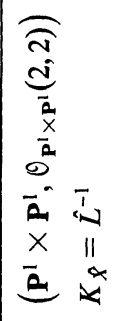 & 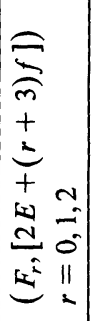 & 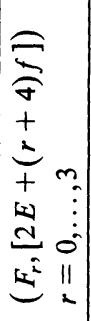 & 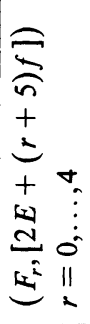 & 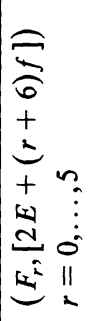 & 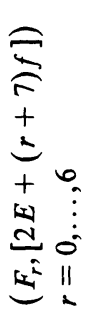 \\
\hline $\mathrm{N}-$ & & & & $\begin{array}{c}a \\
\vdots \\
\vdots \\
n\end{array}$ & $\begin{array}{c}\infty \\
\vdots \\
\vdots \\
n\end{array}$ & $\stackrel{\infty}{\vdots}$ & $\stackrel{\infty}{\vdots}$ & $\begin{array}{c}\infty \\
\vdots \\
\dot{1}\end{array}$ & $\stackrel{\infty}{\vdots}$ & $\begin{array}{c}\infty \\
\vdots \\
\vdots \\
= \\
1\end{array}$ \\
\hline 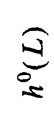 & & & & $\begin{array}{c}\stackrel{0}{\vdots} \\
\vdots \\
\forall\end{array}$ & $\begin{array}{c}a \\
\vdots \\
+\end{array}$ & $\begin{array}{c}\simeq \\
\vdots \\
\vdots\end{array}$ & $\begin{array}{c}\cong \\
\vdots \\
0\end{array}$ & $\stackrel{\infty}{\vdots}$ & $\begin{array}{c}\bar{\tau} \\
\vdots \\
0\end{array}$ & $\begin{array}{c}\text { 志 } \\
\vdots \\
0\end{array}$ \\
\hline$\nabla$ & & & & $\begin{array}{c}a \\
\vdots \\
\dot{n}\end{array}$ & $\begin{array}{c}\infty \\
\vdots \\
\vdots\end{array}$ & $\begin{array}{c}\simeq \\
\vdots \\
\vdots\end{array}$ & $\begin{array}{c}0 \\
\vdots \\
\vdots \\
\end{array}$ & $\begin{array}{c}\text { ণิ } \\
\vdots \\
\infty\end{array}$ & $\begin{array}{c}\text { ন } \\
\vdots \\
\vdots \\
\sigma\end{array}$ & $\begin{array}{c}\stackrel{\infty}{\sim} \\
\vdots \\
\vdots \\
a\end{array}$ \\
\hline $\begin{array}{l}a \\
0 \\
\infty \\
0 \\
0\end{array}$ & & & & & & $N$ & $m$ & $\nabla$ & $n$ & 0 \\
\hline$i$ & & & & 0 & 0 & 0 & 0 & 0 & 0 & 0 \\
\hline 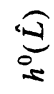 & $m$ & 0 & & 0 & $a$ & $\simeq$ & $\simeq$ & $\infty$ & $\bar{\sim}$ & $\underset{\sim}{\mathbb{N}}$ \\
\hline ¿t & $a$ & $a$ & $\infty$ & $a$ & $\infty$ & $\infty$ & $\infty$ & $\infty$ & $\infty$ & $\infty$ \\
\hline$\nabla$ & - & $\nabla$ & & $a$ & $\infty$ & $\cong$ & $\stackrel{0}{0}$ & ి & $\underset{\sim}{\mathbb{N}}$ & $\stackrel{\infty}{\sim}$ \\
\hline$\infty$ & 0 & 0 & 0 & - & - & $N$ & $m$ & $\nabla$ & $n$ & 0 \\
\hline 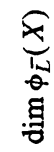 & $\begin{array}{l}8 \\
1\end{array}$ & $\begin{array}{l}8 \\
1\end{array}$ & $\begin{array}{l}8 \\
1\end{array}$ & 0 & 0 & - & - & - & - & - \\
\hline
\end{tabular}




\begin{tabular}{|c|c|c|c|c|c|c|c|c|c|}
\hline $\begin{array}{l}\pi \\
1 \\
1 \\
0\end{array}$ & $r$ & 0 & 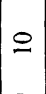 & $a$ & $\infty$ & 으 & $=$ & $\simeq$ & $\underline{2}$ \\
\hline $\begin{array}{l}\tilde{c} \\
1 \\
0 \\
0\end{array}$ & $\infty$ & 0 & $\begin{array}{l}0 \\
1\end{array}$ & 0 & $\stackrel{m}{+}$ & $r$ & $\sigma$ & 0 & $p$ \\
\hline 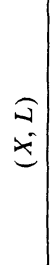 & 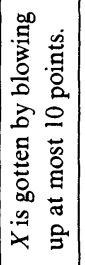 & 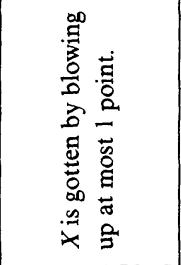 & $\begin{array}{c}x \\
11 \\
x\end{array}$ & $\stackrel{x}{\prime \prime}$ & 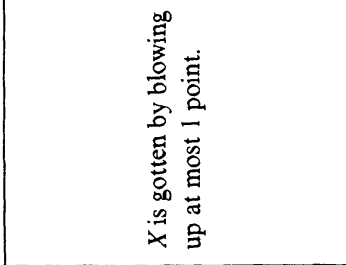 & 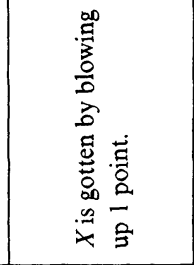 & 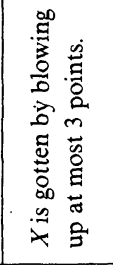 & 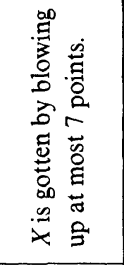 & 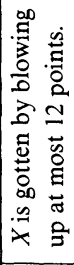 \\
\hline $\begin{array}{l}\widehat{A} \\
\dot{x}\end{array}$ & 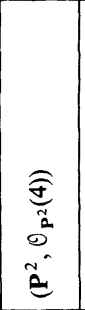 & 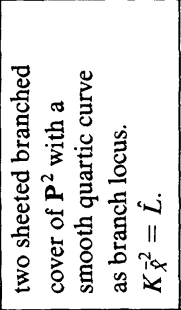 & 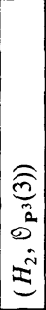 & 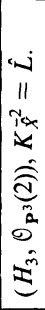 & 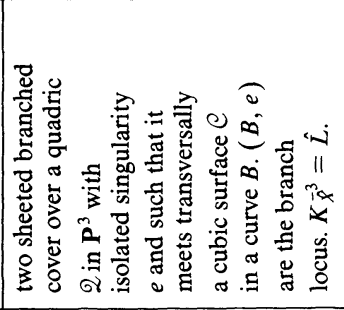 & 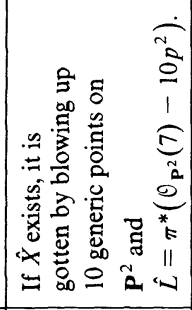 & 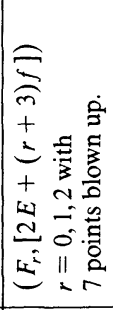 & 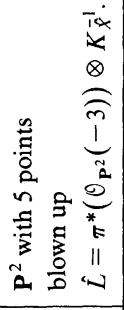 & $\begin{array}{l}\tilde{n} \\
\otimes \\
0 \\
= \\
=\end{array}$ \\
\hline$\tau-$ & $\begin{array}{c}a \\
\vdots \\
i \\
i\end{array}$ & $\cong$ & & & $\overline{0}$ & $\uparrow$ & $\begin{array}{c}- \\
\vdots \\
i\end{array}$ & $\begin{array}{c}\dot{\sigma} \\
\vdots \\
\dot{1}\end{array}$ & $\begin{array}{c}\infty \\
\vdots \\
\dot{1}\end{array}$ \\
\hline $\begin{array}{l}3 \\
\vdots \\
=\end{array}$ & $\begin{array}{c}\simeq \\
\vdots \\
\vdots\end{array}$ & $\hat{\sigma}$ & & & $\hat{\sigma}$ & $n$ & $\begin{array}{c}a \\
\vdots \\
\vdots \\
0\end{array}$ & $\begin{array}{c}m \\
\vdots \\
\vdots\end{array}$ & 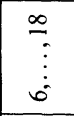 \\
\hline$\nabla$ & $\begin{array}{c}0 \\
\vdots \\
\vdots \\
\sigma\end{array}$ & $\stackrel{\infty}{\sim}$ & & & $\stackrel{a}{\infty}$ & $\infty$ & $\begin{array}{c}\simeq \\
\vdots \\
\vdots\end{array}$ & $\begin{array}{c}0 \\
\vdots \\
\vdots\end{array}$ & $\begin{array}{c}\bar{\lambda} \\
\vdots \\
\vdots\end{array}$ \\
\hline $\begin{array}{l}1 \\
\otimes \\
\otimes \\
\pm \\
\pm \\
0 \\
0\end{array}$ & $m$ & $m$ & $\nabla$ & $\sigma$ & $\sigma$ & $n$ & $n$ & $n$ & $n$ \\
\hline$i$ & - & $N$ & $N$ & $m$ & $\nabla$ & 0 & $n$ & $\nabla$ & $m$ \\
\hline io & 0 & - & 0 & - & $N$ & $m$ & $\mathrm{~N}$ & - & 0 \\
\hline 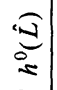 & $\simeq$ & $r$ & 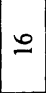 & 으 & $r$ & 0 & $a$ & $m$ & $\stackrel{\infty}{-}$ \\
\hline$\approx$ & $a$ & $N$ & $\infty$ & $m$ & - & $T$ & - & $\sigma$ & $\infty$ \\
\hline$\sigma$ & 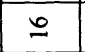 & $\infty$ & $\infty$ & $\simeq$ & $a$ & $a$ & $\simeq$ & $\underline{0}$ & $\bar{\sim}$ \\
\hline$\infty$ & $m$ & $m$ & $\nabla$ & $\theta$ & $\nabla$ & in & $n$ & $n$ & $n$ \\
\hline 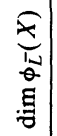 & $N$ & $N$ & $N$ & $N$ & $N$ & $N$ & $N$ & $N$ & $N$ \\
\hline
\end{tabular}




\begin{tabular}{|c|c|c|c|c|c|c|c|}
\hline $\begin{array}{c}\pi \\
1 \\
0 \\
0\end{array}$ & $\cong$ & $\simeq$ & \pm & $\underline{2}$ & $m$ & $\simeq$ & $=$ \\
\hline $\begin{array}{l}\pi \\
1 \\
i\end{array}$ & i & 0 & $\sigma$ & 0 & $r$ & $\infty$ & $\infty$ \\
\hline$\widehat{\jmath}$ & & & & & 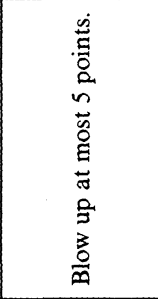 & 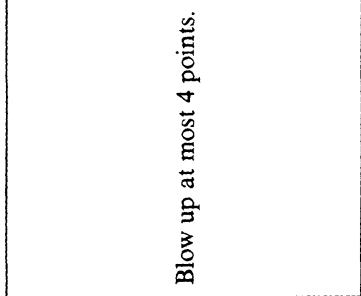 & 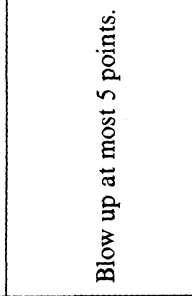 \\
\hline $\begin{array}{l}\widehat{d} \\
\sqrt[x]{ }\end{array}$ & 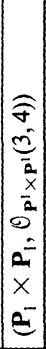 & 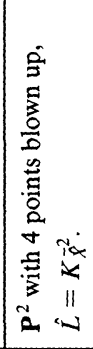 & 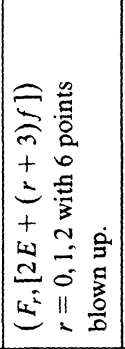 & 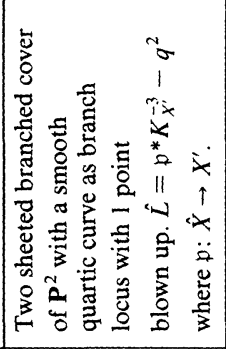 & 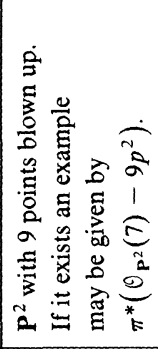 & 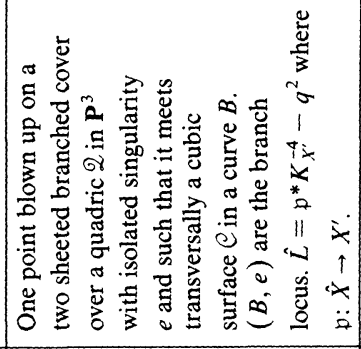 & 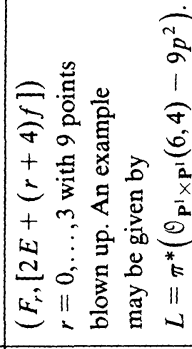 \\
\hline N & $\begin{array}{l}\infty \\
-\end{array}$ & $\begin{array}{l}n \\
\dot{v} \\
m\end{array}$ & $\cong$ & & $\stackrel{0}{i}$ & & \\
\hline$\underset{\Xi}{0}$ & $\begin{array}{c}\stackrel{0}{2} \\
2\end{array}$ & $\begin{array}{c}0 \\
\vdots \\
\square \\
\square\end{array}$ & $\begin{array}{l}\simeq \\
=\end{array}$ & & $\hat{\infty}$ & & \\
\hline$\tau$ & $\begin{array}{l}\vec{d} \\
\vec{\lambda}\end{array}$ & $\begin{array}{c}\stackrel{+}{\vdots} \\
\vdots \\
\infty\end{array}$ & $\begin{array}{l}\simeq \\
\simeq\end{array}$ & & $\frac{m}{\simeq}$ & & \\
\hline $\begin{array}{l}a \\
\otimes \\
x \\
\leq \\
0 \\
=\end{array}$ & 0 & 6 & 0 & 0 & 0 & 0 & 0 \\
\hline$i$ & $\nabla$ & $n$ & 0 & $r$ & $r$ & $\infty$ & $r$ \\
\hline io & 0 & - & $N$ & $m$ & $m$ & $\nabla$ & $m$ \\
\hline$\stackrel{\sqrt{2}}{0}$ & ి. & $\underline{-}$ & $\simeq$ & 으 & $a$ & $\infty$ & $\infty$ \\
\hline$\approx-$ & $\infty$ & $n$ & $N$ & - & 0 & 0 & $T$ \\
\hline$\nabla$ & 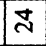 & 구 & 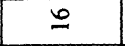 & \pm & $\cong$ & $\simeq$ & $\simeq$ \\
\hline$\infty$ & 0 & 0 & 0 & 0 & 6 & 0 & 0 \\
\hline 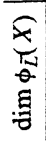 & 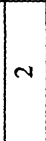 & $N$ & $N$ & $N$ & $N$ & $N$ & $N$ \\
\hline
\end{tabular}




\begin{tabular}{|c|c|c|c|c|c|c|c|}
\hline $\begin{array}{l}u \\
1 \\
0\end{array}$ & $\simeq$ & $=$ & $\simeq$ & $a$ & 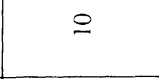 & $=$ & $\underline{\square}$ \\
\hline $\begin{array}{l}\text { 品 } \\
1 \\
i \\
0\end{array}$ & $a$ & 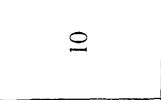 & 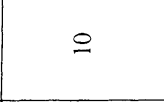 & $=$ & $=$ & $=$ & $p$ \\
\hline $\begin{array}{l}\widehat{A} \\
\dot{ \pm}\end{array}$ & 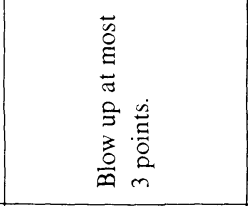 & 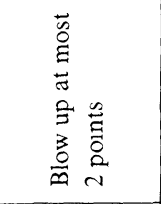 & 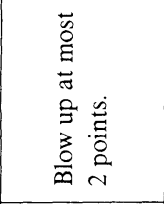 & $\begin{array}{l}x \\
11 \\
x\end{array}$ & $\stackrel{x}{11}$ & $\begin{array}{l}x \\
11 \\
x\end{array}$ & 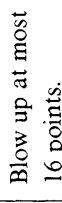 \\
\hline & 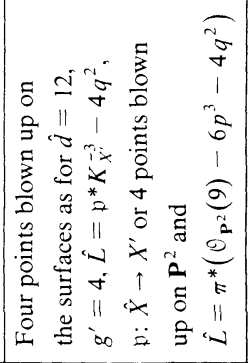 & 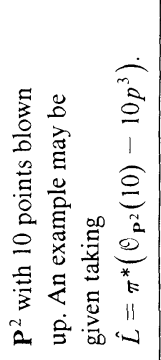 & 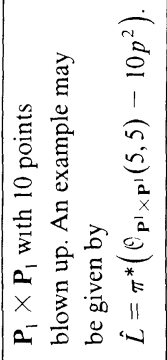 & $\widehat{\mathcal{E}}$ & 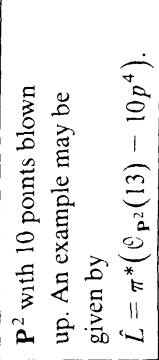 & 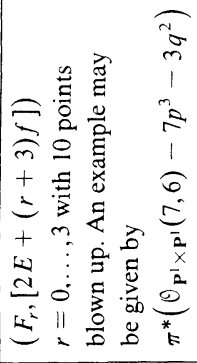 & $\begin{array}{l}\widehat{\widehat{\Xi}} \\
\tilde{\tilde{D}} \\
0 \\
\tilde{E}\end{array}$ \\
\hline ']- & & & & & & & $\vdots$ \\
\hline $\begin{array}{l}3 \\
0 \\
=\end{array}$ & & & & & & & $\begin{array}{c}\bar{\jmath} \\
\vdots \\
0\end{array}$ \\
\hline$\nabla$ & & & & & & & $\begin{array}{c}\approx \\
\vdots \\
\vdots\end{array}$ \\
\hline $\begin{array}{l}a \\
\otimes \\
x \\
\leq \\
= \\
=\end{array}$ & 0 & 0 & 0 & 6 & 0 & 6 & 0 \\
\hline$i$ & $\infty$ & $\sigma$ & $\infty$ & $=$ & 은 & $a$ & $\nabla$ \\
\hline io & $\nabla$ & n & $\nabla$ & $r$ & 0 & $n$ & 0 \\
\hline $\begin{array}{l}\Xi \\
\Xi \\
=\end{array}$ & $r$ & 0 & 0 & 0 & $\begin{array}{l}0 \\
\sim \\
\sim i\end{array}$ & 0 & $\bar{\sim}$ \\
\hline$\tilde{s}^{-}$ & $T$ & $T$ & $\uparrow$ & 0 & $T$ & $\uparrow$ & $a$ \\
\hline ه & $=$ & 으 & 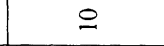 & $a$ & $a$ & $a$ & $\approx$ \\
\hline$+\infty$ & 0 & 0 & 0 & 6 & 6 & 0 & 6 \\
\hline $\begin{array}{l}8 \\
0 \\
0\end{array}$ & $N$ & $N$ & $N$ & $\sim$ & $N$ & $\sim$ & $N$ \\
\hline
\end{tabular}


To prove (2) we use the Algebraic Index Theorem applied to $K_{X}$ and $K_{X} \otimes L$ and the adjunction formula for the line bundle $L$. To prove (3) we use the adjunction formula for the line bundle $K_{X} \otimes L$ and equality (1).

(0.2) THEOREM. Let $X$ be a smooth, connected, projective, rational surface and $L$ a very ample line bundle on it. Let $C \in|L|$. If $g=g(C)=$ $g(L)=0$ then $(X, L)$ is one of the following:

(a) $X=\mathbf{P}^{2}, L=\vartheta_{\mathbf{P}^{2}}(1)$ or $L=\vartheta_{\mathbf{P}^{2}}(2)$.

(b) $X=F_{r}, L=\left[E_{\infty}+k f\right], k \geq r+1$.

If $g=1$ then $X$ is one of the following:

(c) $X=\mathbf{P}^{2}$ with at most six points blown up.

(d) $X=\mathbf{P}^{1} \times \mathbf{P}^{1}$ and $L \cdot L=8$.

Proof. If $g(C)=0$, the result follows by Del Pezzo's Theorem (0.6) Now suppose $g(C)=1$. Since by the First Lefschetz Theorem $[\mathbf{A}+\mathbf{F}]$ and [Bo], $h^{1,0}(X) \leq g(C)$, we have to examine two cases:

(i) $g(C)=1, h^{1,0}(X)=0$,

and

(ii) $g(C)=1, h^{1,0}(X)=1$.

In case (i), since $g(C)=1, C$ is an elliptic curve. Therefore $K_{C}$ is trivial, which implies $K_{X} \otimes L$ is trivial. Thus $K_{X} \approx L^{-1}$. Since $L^{-1}$ is a negative line bundle, it follows that $K_{X}$ is a negative line bundle, so $X$ is a ruled rational surface. Moreover, by Serre duality [Ha, pg 239],

$$
h^{1}\left(\left.L\right|_{C}\right)=h^{0}\left(\left.L^{-1}\right|_{C} \otimes K_{C}\right)=h^{0}\left(\left.L^{-1}\right|_{C}\right)=0
$$

because $K_{C}$ is trivial and $\left.L^{-1}\right|_{C}$ is a negative line bundle. Since $h^{1}\left(\left.L\right|_{C}\right)=0$, by the Riemann-Roch Theorem [Ha, pg 295],

$$
h^{0}\left(\left.L\right|_{C}\right)=d-g(C)+1=d,
$$

where $d=L \cdot L$. Now using the long cohomology sequence associated to the short exact sequence

$$
\left.0 \rightarrow \Theta_{X} \rightarrow L \rightarrow L\right|_{C} \rightarrow 0,
$$

we obtain that $h^{0}(L)=d+1$. Hence $X$ is a ruled, rational surface of degree $d$ in $\mathbf{P}^{d}$. Then by [N, pg 366], $X$ has to be either (c) or (d). Moreover, by [N], since $X$ is non-singular, if $\mathscr{P}_{i}$ denote the points that we blow up on $\mathbf{P}^{2}$ to get $X$, then the $\mathscr{P}_{i}$ have to satisfy the following two conditions:

(1) any three points among the $\mathscr{P}_{l}$ are not colinear;

(2) there is no conic carrying six of the $\mathscr{P}_{l}$. 
In case (ii), since $g(C)=1=h^{1,0}(X)$, again using Del Pezzo's Theorem, we have that $X$ is a $\mathbf{P}^{1}$-bundle over a curve $\Gamma$ and smooth $C \in|L|$ are sections. Since $C$ is a section, $g(\Gamma)=g(C)=1$, i.e. $X$ is a $\mathbf{P}^{1}$-bundle over an elliptic curve.

(0.3) Proposition. Let $X$ be a smooth, connected, projective surface

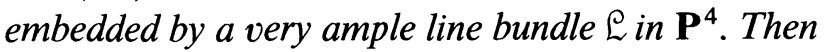

$(0.3 .1) \mathcal{L} \cdot \mathcal{L}(\mathcal{L} \cdot \mathcal{L}-5)-10(g(\mathcal{L})-1)+12 \chi\left(\Theta_{X}\right)=2 K_{X} \cdot K_{X}$.

Proof. See [Ha, pg 434].

(0.4) Castelnuovo's Inequality [Ba, pg $234 \mathrm{ff} ; \mathbf{G}+\mathbf{H}$ ]: If $\mathrm{C}$ is an irreducible curve embedded in $\mathbf{P}^{l-1}$ and $C$ belongs to no linear hyperplane $\mathbf{P}^{l-2}$, then with $d$ the degree of $C$ and $g$ the genus:

$$
g \leq\left[\frac{d-2}{l-2}\right]\left(d-l+1-\left[\frac{d-l}{l-2}\right]\left(\frac{l-2}{2}\right)\right),
$$

where [ ] is the least integer function.

(0.5) Clifford's TheOrem. Let $L$ be an invertible sheaf on a curve $C$ such that

$$
0 \leq \operatorname{deg} L \leq 2 g(C)-2 \text {. }
$$

Then

$$
2\left(h^{0}(L)-1\right) \leq \operatorname{deg} L
$$

and equality holds only in the following cases:

(i) $L=\vartheta_{C}, L=K_{C}$;

(ii) $C$ is hyperelliptic.

[Sa, pg 158].

(0.6) Del Pezzo's Theorem. Let $L$ be a very ample line bundle on a surface $X$. Then $g(L)=h^{1,0}(X)$ if and only if

(a) $X=\mathbf{P}^{2}$ and $L=\theta_{\mathbf{P}^{2}}(1)$ or $\theta_{\mathbf{P}^{2}}(2)$.

(b) $X$ is a $\mathbf{P}^{1}$-bundle over a curve and smooth $C \in|L|$ are sections. Moreover, if $h^{1,0}(X)=0$ then in case (b) $X$ is a Hirzebruch surface.

For a modern proof see [Fu] or, for example, [So $\mathbf{S}_{\mathbf{1}},(0.6 .1) \mathrm{pg}$ 19]. 
1. The case of $h^{0}(L)=3,4$.

Let $X$ be a smooth, connected, projective, rational surface and $L$ a very ample line bundle on it. If $h^{0}(L)=3$ then

$$
(X, L)=\left(\mathbf{P}^{2}, \Theta_{\mathbf{P}^{2}}(1)\right) \text {. }
$$

If $h^{0}(L)=4$ then

$$
(X, L)=\left(F_{0}, E \otimes f\right) .
$$

The proof follows from the following propositions.

(1.1) Proposition. Let $X$ and $L$ be as in the above theorem but without assuming that $X$ is rational. If $h^{0}(L)=3$ then $g=0$ and $(X, L)=$ $\left(\mathbf{P}^{2}, \Theta_{\mathbf{P}^{2}}(1)\right)$. If $h^{0}(L)=4$ then $g=0,3,6$ and $h^{1,0}(X)=0$.

Proof. If $h^{0}(L)=3$ we see trivially that the statement is true. If $h^{0}(L)=4$ then $X$ is a hypersurface in $\mathbf{P}^{3}$; hence by the First Lefschetz Theorem $[\mathbf{A}+\mathbf{F}], h^{1,0}(X)=0$. Since $C$ is embedded in $\mathbf{P}^{2}$ we get $g=0$, 1, 3, 6 .

Suppose $h^{0}(L)=4$ and $g=0$. By Del Pezzo's Theorem [N], $X$ is a Hirzebruch surface. Let $X=F_{r}, L=\left[E_{\infty}+k f\right] k \geq r+1$. By the Riemann-Roch Theorem and the long cohomology sequence of the short exact sequence

$$
\left.0 \rightarrow \theta_{X} \rightarrow L \rightarrow L\right|_{C} \rightarrow 0,
$$

we get $d=2$. Thus $2=\left(E_{\infty}+k f\right) \cdot\left(E_{\infty}+k f\right)$, which implies $r=0$, i.e. $2=d=2 k \Rightarrow k=1$. Therefore $(X, L)=\left(F_{0}, E_{\infty}+f\right)$. Now suppose $h^{0}(L)=4$ and $g=1$. By Theorem (0.2) either $X$ is $\mathbf{P}^{2}$ with at most six points blown up or $X=\mathbf{P}^{1} \times \mathbf{P}^{1}$ and $d=8$. Since $h^{0}(L)=d+1$, see Theorem (0.2); thus $d=3$, which excludes the case $X=\mathbf{P}^{1} \times \mathbf{P}^{1}$. Thus $X$ has to be a blow up of $\mathbf{P}^{2}$. By $K_{X}=L^{-1}$ we obtain $c_{1}^{2}=-3$, which contradicts the fact that $K_{\mathbf{P}^{2}} \cdot K_{\mathbf{P}^{2}}=9$, and we can blow up at most six points. Therefore if $h^{0}(L)=4$ then $g=0,3,6$.

(1.2) Proposition. Let $(X, L)$ be as in the previous proposition. Let $h^{0}(L)=4$. If $g=3$ then $(X, L)=\left(H_{4}, \Theta_{\mathbf{P}^{3}}(1)\right)$, where $H_{4}$ is a hypersurface of degree 4 in $\mathbf{P}^{3}, h^{1,0}(X)=0, h^{2,0}(X)=1$, and $L \cdot L=4$. If $g=6$, then $(X, L)=\left(H_{5}, \vartheta_{\mathbf{P}^{3}}(1)\right)$, where $H_{5}$ is a hypersurface in $\mathbf{P}^{3}$, $h^{1,0}(X)=0, h^{2,0}(X)=4$ and $L \cdot L=5$. Moreover, in the first case, $K_{H_{4}}$ is trivial, and in the second case, $K_{H_{5}}=L=\vartheta_{\mathbf{P}^{3}}(1)$. 
Proof. We will first study the case in which $g=3$. Let $\delta$ be the degree of $C$. Since $C$ is embedded in $\mathbf{P}^{2}$,

$$
g=\frac{1}{2}(\delta-1)(\delta-2),
$$

which gives $\delta=4$, i.e. $C$ is a curve of degree four in $\mathbf{P}^{2}$. It follows that $X$ is a degree four surface in $\mathbf{P}^{3}$. Hence

$$
(X, L)=\left(H_{4},\left.\theta_{\mathbf{P}^{3}}(1)\right|_{H_{4}}\right) .
$$

Since $K_{H_{4}}=\theta_{\mathbf{P}^{3}}(d-4), K_{H_{4}}$ is trivial, by the adjunction formula, so $h^{0}\left(K_{H_{4}}\right)=1$, which implies $h^{1,0}(X)=0$ and $h^{2,0}(X)=1$. Moreover, $\operatorname{deg}\left(\left.\theta_{\mathbf{P}^{3}}(1)\right|_{H_{4}}\right)=4$.

$$
\begin{aligned}
\operatorname{deg}\left(\left.\vartheta_{\mathbf{P}^{3}}(1)\right|_{H_{4}}\right) & =\left.\left.\vartheta_{\mathbf{P}^{3}}(1)\right|_{H_{4}} \cdot \vartheta_{\mathbf{P}^{3}}(1)\right|_{H_{4}} \\
& =\vartheta_{\mathbf{P}^{3}}(1) \cdot \vartheta_{\mathbf{P}^{3}}(1) \cdot \vartheta_{H_{4}}=4 .
\end{aligned}
$$

Now consider the case $g=6$. As before we see that $X$ is a degree five hypersurface in $\mathbf{P}^{3}$. Thus

$$
(X, L)=\left(H_{5},\left.\vartheta_{\mathbf{P}^{3}}(1)\right|_{H_{5}}\right) .
$$

In this case $K_{H_{5}}=\vartheta_{\mathbf{P}^{3}}(1)$, hence $L=K_{H_{5}}$, i.e. $H_{5}$ is embedded in $\mathbf{P}^{3}$ by the canonical line bundle. So $K_{H_{5}} \otimes L=\vartheta_{\mathbf{P}^{3}}(2)$. Moreover, $h^{1,0}(X)=0$, $h^{2,0}(X)=4$ and $\operatorname{deg}\left(\left.\vartheta_{\mathbf{P}^{3}}(1)\right|_{\mathrm{H}_{5}}\right)=5$.

2. The case of $\operatorname{dim} \phi_{\bar{L}}=1$. Under the hypothesis that $\operatorname{dim} \phi_{\bar{L}}(X)$ $=1$, by [So, (2.1) pg 390], if $\phi_{\bar{L}}=r \circ s$ is the Remmert-Stein factorization of $\phi_{\bar{L}}$, then $X \stackrel{r}{\rightarrow} Y \stackrel{s}{\rightarrow} \mathbf{P}$, where $X$ is a $\mathbf{P}^{1}$-bundle over $Y$ which is embedded in $\mathbf{P}$. Moreover,

$$
c_{1}^{2}+4 g-4=d .
$$

(2.1) LemMa. Let $X$ be as before. Then $h^{0}(L) \geq 6$ unless

$$
g=2, \quad d=5, \quad c_{1}^{2}=1 .
$$

Proof. Since we may assume $h^{0}(L) \geq 5$, in order to prove the lemma we assume $h^{0}(L)=5$. Now using Proposition (0.3) and (2.0.1) we see that the following invariants are the only ones possible:

(1) $g=2, d=2, c_{1}^{2}=-2$;

(2) $g=2, d=5, c_{1}^{2}=1$;

(3) $g=4, d=1, c_{1}^{2}=-11$;

(4) $g=4, d=6, c_{1}^{2}=-6$.

Cases (1) and (3) contradict Castelnuovo's inequality (0.4) and case (4), since $h^{0}\left(\left.L\right|_{C}\right)=4=g$, implies, by [So, (0.9.6) pg 382], that $K_{X}$ is trivial, which gives a contradiction. 
Now we assume $h^{0}(L) \geq 6$. Consider the long cohomology sequence associated to the short exact sequence

$$
\left.0 \rightarrow \hat{\theta}_{X} \rightarrow L \rightarrow L\right|_{C} \rightarrow 0 .
$$

Since $h^{0}(L) \geq 6$, then $h^{0}\left(\left.L\right|_{C}\right) \geq 5$ and $\left.\left.L\right|_{C} \cdot L\right|_{C} \geq 2 g-2$ except when $g=6, d=9$ and $h^{0}(L)=6$. In fact if $\left.\left.L\right|_{C} \cdot L\right|_{C} \leq 2 g-3$, by Clifford's Theorem (0.5)

$$
h^{0}\left(\left.L\right|_{C}\right) \leq \frac{2 g-3}{2}+1 \leq g-1 .
$$

Let $g=6$. Then $h^{0}\left(\left.L\right|_{C}\right)=5$ and by Castelnuovo's inequality $d \geq 9$. Hence

$$
9 \leq d \leq 2 g-3=9 .
$$

Therefore $d=9$. Now assume that $L \cdot L \geq 2 g-2$. Using the fact that for a birational ruled surface with $h^{1,0}(X) \neq g$,

$$
\frac{L \cdot L}{8}+h^{1,0}(X) \leq \frac{g+1}{2},
$$

which follows by [So, (2.1) pg 390], we obtain

$$
L \cdot L \leq 4(g+1) \text {. }
$$

By Castelnuovo's inequality and (2.0.1), it follows that if $X$ is birational to a Hirzebruch surface then the pair $(X, L)$ has to satisfy the following invariants:

(1) $g=2,5 \leq d \leq 12,5 \leq h^{0}(L) \leq 12,1 \leq c_{1}^{2} \leq 8$;

(2) $g=3,7 \leq d \leq 16,6 \leq h^{0}(L) \leq 15,-1 \leq c_{1}^{2} \leq 8$;

(3) $g=4,8 \leq d \leq 20,6 \leq h^{0}(L) \leq 18,-4 \leq c_{1}^{2} \leq 8$;

(4) $g=5,8 \leq d \leq 24,6 \leq h^{0}(L) \leq 21,-8 \leq c_{1}^{2} \leq 8$

(5) $g=6,9 \leq d \leq 28,6 \leq h^{0}(L) \leq 24,-11 \leq c_{1}^{2} \leq 8$.

Consider now the case in which $g=5$. If we assume $d=8$, then by Castelnuovo's inequality $h^{0}(L)=5$ or 6 . Since in the case in which $h^{0}(L)=6, K_{X}$ is trivial, see [So, (0.9.6) pg 382], we have that $h^{0}(L)=5$ and, using Proposition (0.3), we obtain $c_{1}^{2}=-2$. But the invariants $d=8$ and $c_{1}^{2}=-2$ contradict the fact that if $X$ is a minimal model then $d=24$, $c_{1}^{2}=8$. Therefore $d \neq 8$. Since if $X=\mathbf{P}^{2}, \operatorname{dim} \phi_{L}^{-}(X)=2$, we can state the following theorem.

(2.2) THEOREM. Let $X$ be a smooth, connected, projective, rational surface such that $\operatorname{dim} \phi_{\bar{L}}(X)=1$. Denote by $\hat{X}$ a minimal model and $\hat{L}$ the relative line bundle. Then

$$
(\hat{X}, \hat{L})=\left(F_{r}, 2 E+(g+r+1) f\right), \quad r=0, \ldots, g .
$$


For $(X, L)$ we have obtained the following sets of invariants:

(1) $g=2,5 \leq d \leq 12,5 \leq h^{0}(L) \leq 12,1 \leq c_{1}^{2} \leq 8$, at most seven points blown up

(2) $g=3,7 \leq d \leq 16,6 \leq h^{0}(L) \leq 15,-1 \leq c_{1}^{2} \leq 8$, at most nine points blown up

(3) $g=4,8 \leq d \leq 20,6 \leq h^{0}(L) \leq 18,-4 \leq c_{1}^{2} \leq 8$, at most twelve points blown up

(4) $g=5,9 \leq d \leq 24,6 \leq h^{0}(L) \leq 21,-8 \leq c_{1}^{2} \leq 8$, at most fifteen points blown up

(5) $g=6,9 \leq d \leq 28,6 \leq h^{0}(L) \leq 24,-11 \leq c_{1}^{2} \leq 8$, at most nineteen points blown up.

Thus we get the table on page 95 .

3. The case of $\operatorname{dim} \phi_{\bar{L}}(X)=2$. Under the hypothesis that $\operatorname{dim} \phi_{\bar{L}}(X)=2$, A. J. Sommese has proved in [So, (2.3) pg 392] that, if $\phi_{\bar{L}}=r \circ s$ is the Remmert-Stein factorization of $\phi_{\bar{L}}$, then $X \stackrel{r}{\rightarrow} \hat{X} \stackrel{s}{\rightarrow} \mathbf{P}^{n}$, where $r: X \rightarrow \hat{X}$ expresses $X$ as $\hat{X}$ with a finite set $F$ of points blown up. Further,

$$
K_{X} \otimes L=r^{*}\left(K_{\hat{X}} \otimes \hat{L}\right)
$$

where $\hat{L}$ is an ample line bundle on $\hat{X}$ with

$$
L=\left(r^{*} \hat{L}\right) \otimes\left[r^{-1} F\right]^{-1}
$$

In particular, $s=\phi_{L^{\prime}}$. Moreover if $H^{\mathrm{l}}(X, L)=0$ then $\hat{L}$ is very ample, and since $h^{1,0}(X)=0, s$ is an embedding unless there is a smooth hyperelliptic $C \in|L|$. He has also proved in (3.1) pg 395 that this last situation can only happen when:

(3.0.1) $\hat{X}$ is a two-sheeted branched cover of $\mathbf{P}^{2}, p: \hat{X} \rightarrow \mathbf{P}^{2}$ with a smooth quartic curve as branch locus $B$. Moreover, $L=\left[p^{-1}(B)\right]$ is a very ample line bundle on $\hat{X}, g=3$ and $\phi_{\bar{L}}$ is simply p. Further, $\operatorname{dim} h^{0}(\hat{L})=7$ and $\hat{d}=8$.

(3.0.2) $\hat{X}$ is a smooth, connected, projective surface which is a 2-sheeted branched cover $p: \hat{X} \rightarrow \mathcal{2}$, where 2 is a quadric in $\mathbf{P}^{3}$ with an isolated singularity $e$ and such that it meets transversally a cubic surface $C_{\text {in }}$ a curve $B$. $\hat{X}$ is such that $B$ and $e$ are its branch locus. In this case $g=4$, $\operatorname{dim} h^{0}(\hat{L})=7$ and $\hat{d}=9$. 
(3.1) LeMma. Let $X$ be a smooth, connected, projective, rational surface such that $\operatorname{dim} \phi_{\bar{L}}^{-}(X)=2$. Then $h^{0}\left(K_{X} \otimes L\right)=g$.

Proof. Use the long cohomology sequence of

$$
0 \rightarrow K_{X} \rightarrow K_{X} \otimes L \rightarrow K_{C} \rightarrow 0 \text {. }
$$

(3.1') Corollary. Let $X$ be as above. Then $g \neq 2$.

Proof. Use Lemma (3.1).

By Castelnuovo's inequality and (2.1.3) it follows that:

$$
\begin{array}{lll}
\text { if } g=6 & \text { then } \quad 7 \leq d \leq 28 ; \\
\text { if } g=5 & \text { then } 7 \leq d \leq 24 ; \\
\text { if } g=4 & \text { then } 6 \leq d \leq 20 ; \\
\text { if } g=3 & \text { then } 6 \leq d \leq 16
\end{array}
$$

(3.2) TheOREM. Let $X$ be a smooth, connected, projective, rational surface such that $\operatorname{dim} \phi_{\bar{L}}(X)=2$. Suppose $(\hat{X}, \hat{L})$ is a minimal pair. If $g=3$ then either $(\hat{X}, \hat{L})=\left(\mathbf{P}^{2}, \hat{\theta}_{\mathbf{P}^{2}}(4)\right)$ or $(\hat{X}, \hat{L})$ is as in (3.0.1). If $g(L)=4$ then the following cases are possible:

(i) $(\hat{X}, \hat{L})=\left(H_{3}, \theta_{\mathbf{p}^{3}}(2)\right)$;

(ii) $(\hat{X}, \hat{L})=\left(H_{2}, \hat{\theta}_{\mathbf{P}^{3}}(3)\right)$;

(iii) $(\hat{X}, \hat{L})$ is as in $(3.0 .2)$.

Proof. Let $g=3$. By Lemma (3.1) we know that the adjunction map surjects onto $\mathbf{P}^{2}$. By the long cohomology sequence of (2.1.2) it follows that $h^{1}(L)=0$. Hence by [So, (2.4) pg 393], $\hat{L}$ is very ample. If there exists a $C \in|L|$ which is hyperelliptic, again by [So, (3.1) pg 395], $X$ is as in (3.0.2). So we can suppose there is no such $C$. Since by [So, (3.1) pg 395], $\phi_{L^{\prime}}$ is an embedding, we have that $\hat{X}=\mathbf{P}^{2}$ and

$$
K_{\hat{X}} \otimes \hat{L}=K_{\mathbf{P}^{2}} \otimes \hat{L}=\mathcal{O}_{\mathbf{P}^{2}}(-3) \otimes \hat{L},
$$

which gives $\hat{L}=\hat{O}_{\mathbf{P}^{2}}(4)$. Thus $\hat{d}=16, \hat{c}_{1}^{2}=9$ and $h^{0}(\hat{L})=15$.

Now suppose $g=4$. Assume first that

$$
d=6=2 g-2 \text {. }
$$

By the Riemann-Roch Theorem

$$
h^{0}\left(\left.L\right|_{C}\right)=3+h^{1}\left(\left.L\right|_{C}\right)
$$

and by Clifford's Theorem

$$
h^{0}\left(\left.L\right|_{C}\right) \leq 4
$$


Since in the case in which $h^{0}\left(\left.L\right|_{C}\right)=4$, by [So, (0.9.6) pg 382], $K_{X}$ is trivial, it follows that $h^{0}\left(\left.L\right|_{C}\right)=3$ and $h^{1}\left(\left.L\right|_{C}\right)=0$. Thus by the long cohomology sequence of (2.1.2), $h^{1}(L)=0$, and by [So, (2.4) pg 393] we obtain that $\hat{L}$ is very ample. If $d \geq 7$, again by (2.1.2), $h^{1}(L)=0$ and $\hat{L}$ is very ample. If there is a $C \in|L|$ which is hyperelliptic, again by [So, (3.1) pg 395], we have that $(\hat{X}, \hat{L})$ is as in (3.0.2). Thus we can suppose there is no such $C$ in $|L|$. Again by [So, (3.1) pg 395], since $h^{1,0}(X)=0, \phi_{L^{\prime}}$ gives an embedding in $\mathbf{P}^{3}$ i.e. $\hat{X}$ is a hypersurface in $\mathbf{P}^{3}$. Denote $\hat{X}$ by $H_{\delta}$. $K_{H_{\delta}}=\vartheta_{\mathbf{P}^{3}}(\delta-4)$ and $K_{\hat{X}} \otimes \hat{L}=\vartheta_{\mathbf{P}^{3}}(1)$. So

$$
\vartheta_{\mathbf{P}^{3}}(\delta-4) \otimes \hat{L}=\vartheta_{\mathbf{P}^{3}}(1) \text {. }
$$

Let $\hat{L}=\vartheta_{\mathbf{P}^{3}}(k)$. Then

$$
\mathcal{O}_{\mathbf{P}^{3}}(\delta+k-4)=\mathcal{O}_{\mathbf{P}^{3}}(1) .
$$

Therefore

$$
\hat{L}=\left.\theta_{\mathbf{P}^{3}}(5-\delta)\right|_{\hat{X}}
$$

and since $\hat{L}$ is very ample, $\delta \leq 4$. Thus

$$
7 \leq \hat{d}=\delta(5-\delta)^{2} \text {. }
$$

Let $\delta=0$. Then $\hat{d}=0$, which gives a contradiction. Let $\delta=1$. Then

$$
\hat{d}=(5-\delta)^{2} \cdot \delta=16 .
$$

Since $\delta=1 \operatorname{deg}(\hat{X})=1, \hat{X}$ is mapped into a linear subspace, i.e. $\hat{X} \hookrightarrow \mathbf{P}^{2}$. Thus $h^{0}\left(K_{\hat{X}} \otimes \hat{L}\right)=3$, which gives a contradiction since $h^{0}\left(K_{\hat{X}} \otimes \hat{L}\right)=4$ by Lemma (3.1). Let $\delta=2$. Then $\hat{d}=18, \hat{c}_{1}^{2}=8, h^{0}(\hat{L})=16$. Let $\delta=3$. Then $\hat{d}=12, \hat{c}_{1}^{2}=3, h^{0}(\hat{L})=10$. So if $\delta=2$ then $\hat{X}$ is a quadric in $\mathbf{P}^{3}$ and $\hat{L}=\mathcal{\vartheta}_{\mathbf{P}^{3}}(3)$, i.e. (ii). If $\delta=3$ then $\hat{X}$ is a cubic in $\mathbf{P}^{3}$ and $\hat{L}=\mathcal{\vartheta}_{\mathbf{P}^{3}}(2)$, i.e. (i).

(3.3) TheOrem. Let $(X, L)$ be as in the previous theorem. If $X$ is a minimal model relative to $L$ then $X$ is given as in the above theorem. If $X$ is not a minimal model relative to $L$ and $g=3$ then $X$ is gotten by blowing up at most ten points on $\mathbf{P}^{2}$. If $g=4$ then $X$ is gotten by blowing up one point on the surface in (3.0.2).

Proof. Let $g=3$. We have seen that $6 \leq d \leq 16$. In the case in which there exists a hyperelliptic hyperplane section, we can assume $d=6,7$. If $d=6, c_{1}^{2}=0$ and $h^{0}(L)=5$. Thus using $(0.3 .1)$ we get a contradiction. If $d=7, c_{1}^{2}=1$ and $h^{0}(L)=5,6$. Since in the case in which $h^{0}(L)=5$ we get a contradiction as above, it follows that $h^{0}(L)=6$. Thus, in this case, 
$X$ is gotten by blowing up one point on the relative minimal model. Now consider the case when $C$ is not hyperelliptic. Since $6 \leq d \leq 16, X$ can be gotten by blowing up at most ten points on $\mathbf{P}^{2}$. In the same way we get the statement for $g=4$.

We will use Proposition (0.1) to compute the values of $d^{\prime}$ and $g^{\prime}$. See the table on page 96.

Now we study the case $g=5$. In this case $h^{0}\left(L^{\prime}\right)=5$. Thus $\hat{X}$ is embedded in $\mathbf{P}^{4}$ by $L^{\prime}$ and by Proposition (0.3) we get

$$
d^{\prime}\left(d^{\prime}-5\right)-10\left(g^{\prime}-1\right)+12=2 c_{1}^{\prime 2} .
$$

Since $h^{0}(L) \geq 5$ by Castelnuovo's inequality, $d \geq 7$. If $d=7$, again by Castelnuovo's inequality, $h^{0}(L)=5$. Then applying Proposition (0.3), we obtain $c_{1}^{2}=-7$. Let $(\hat{X}, \hat{L})$ be a minimal pair. Then $\hat{d} \geq 7$. If $\hat{d}=d=7$, using (3.3.1) we get a contradiction. Therefore $\hat{d} \geq 8$. Let $\hat{d}=8$. By Clifford's Theorem and the fact that $h^{0}(\hat{L})=6$ implies $K_{\hat{X}}$ is trivial, we have $h^{0}(\hat{L}) \leq 6$. Thus $h^{0}(\hat{L})=5$. Note that in this case we don't know if $\hat{L}$ is very ample. Since $d=7, c_{1}^{2}=-7$ and $\hat{d}=8$ then $\hat{c}_{1}^{2}=-6$. Since $\hat{X}$ is a minimal model relative to $L$ and $L^{\prime}$ is spanned by [So, (1.5) pg 387], then $K_{\hat{X}} \cdot K_{\hat{X}}=\hat{c}_{1}^{2}=c_{1}^{\prime 2}=K_{X^{\prime}} \cdot K_{X^{\prime}}$, where $X^{\prime}$ is $\hat{X}$ considered in respect to $L^{\prime}$. Thus $c_{1}^{\prime 2}=-6$ and we get a contradiction since (3.3.1) has no solution. Hence $\hat{d} \geq 9$ and $c_{1}^{\prime 2}=\hat{c}_{1}^{2} \geq 5$. Now using Proposition (0.1) we get $d^{\prime} \leq 7$. Since $d^{\prime} \geq g+h^{2,0}-h^{1,0}-2$, it follows that

$$
3 \leq d^{\prime} \leq 7 \text {. }
$$

By Proposition (0.1) and (3.3.1) we get:

(a) $d^{\prime}=7, g^{\prime}=4, c_{1}^{\prime 2}=-2$;

(b) $d^{\prime}=6, g^{\prime}=3, c_{1}^{\prime 2}=-1$;

(c) $d^{\prime}=5, g^{\prime}=2, c_{1}^{\prime 2}=1$;

(d) $d^{\prime}=4, g^{\prime}=1, c_{1}^{\prime 2}=4$

(e) $d^{\prime}=3, g^{\prime}=0, c_{1}^{\prime 2}=8$.

Because $c_{1}^{\prime 2}$ has to be greater than or equal to five, only the last case is possible. Using Proposition (0.1) and from the short exact sequence

$$
\left.0 \rightarrow \theta_{\hat{X}} \rightarrow \hat{L} \rightarrow \hat{L}\right|_{\hat{C}} \rightarrow 0
$$

we obtain $\hat{d}=21$ and $h^{0}(\hat{L})=18$. Since $\hat{c}_{1}^{2}=c_{1}^{\prime 2}=8, \hat{d}=21$ and $d=7$, we must have $c_{1}^{2}=-6$ which contradicts the fact that $c_{1}^{2}=-7$. Therefore $d \neq 7$.

Now assume $d=8$. By Castelnuovo's inequality $h^{0}(L)=5$, 6. If $h^{0}(L)=6$, by [So, (0.9.6) pg 382], $K_{X}$ is trivial, thus $h^{0}(L)=5$. By the Riemann-Roch Theorem and (2.1.2) it follows that $h^{1}(L)=0$ which, by 
[So, (2.4) pg 393], implies that $\hat{L}$ is very ample, and by Proposition (0.3) we get $c_{1}^{2}=-2$. By Proposition (0.1) we have $d^{\prime}=6$ and $g^{\prime}=3$. Since $\hat{c}_{1}^{2}=c_{1}^{\prime 2}$, by $(3.3 .1)$ we get $\hat{c}_{1}^{2}=-1$. Therefore $\hat{d}=9$ and $h^{0}(\hat{L})=5$. We have obtained the following invariants:

$$
\begin{aligned}
& \hat{d}=9, \quad h^{0}(\hat{L})=6, \quad \hat{c}_{1}^{2}=-1, \quad d^{\prime}=6, \\
& g^{\prime}=3, \quad d=8, \quad h^{0}(L)=5, \quad c_{1}^{2}=-2 .
\end{aligned}
$$

Now we look at the table for the genus three surfaces. Since $d^{\prime}-\hat{c}_{1}^{2}=6$ $+1=7$, if it exists, $\hat{X}$ has to be $\mathbf{P}^{2}$ with ten points blown up. Because the degree has to be six and the genus has to be three, an example of $\hat{X}$ may be given by $\hat{L}=\pi^{*}\left(\Theta_{\mathbf{P}^{2}}(7)-10 p^{2}\right)$, where $\pi: \hat{X} \rightarrow \mathbf{P}^{2}$ is the blow up map and by $10 p^{2}$ we mean ten generic points each considered twice. Unfortunately we don't know if $\pi^{*}\left(\vartheta_{\mathbf{P}^{2}}(7)-10 p^{2}\right)$ is very ample. Let $d \geq 9$. Then $h^{1}(L)=0$ and by [So, (2.4) pg 393], $\hat{L}$ is very ample. By the Riemann-Roch Theorem and

$$
\left.0 \rightarrow \Theta_{X} \rightarrow L \rightarrow L\right|_{C} \rightarrow 0,
$$

it follows that $h^{0}(L)=6, \hat{d} \geq 9, h^{0}(\hat{L}) \geq 6$. By Proposition $(0.1), d^{\prime} \leq 7$. Moreover,

$$
d^{\prime} \geq g+h^{2,0}-h^{1,0}-2 .
$$

Thus $3 \leq d^{\prime} \leq 7$. By Proposition (0.1) and (3.3.1) we get

\begin{tabular}{|c|r|r|r|l|}
\hline$d^{\prime}$ & $g^{\prime}$ & $\hat{c}_{1}^{2}$ & $\hat{d}$ & \\
\hline 7 & 4 & -2 & 7 & contradicts $\hat{d} \geq 9$ \\
\hline 6 & 3 & -1 & 9 & $\begin{array}{l}\text { same relative minimal model } \\
\text { as in the earlier case }\end{array}$ \\
\hline 5 & 2 & 1 & 12 & \\
\hline 4 & 1 & 4 & 16 & \\
\hline 3 & 0 & 8 & 21 & \\
\hline
\end{tabular}

In the case in which $d^{\prime}=3, g^{\prime}=0, \hat{c}_{1}^{2}=\hat{c}_{1}^{\prime 2}=8, \hat{d}=21$, by Theorem (0.2), $\hat{X}$ is a Hirzebruch surface, and by the short exact sequence

$$
\left.0 \rightarrow \Theta_{\hat{X}} \rightarrow \hat{L} \rightarrow \hat{L}\right|_{\hat{C}} \rightarrow 0
$$

we get $h^{0}(\hat{L})=18$. So $\hat{X}=F_{r}, L^{\prime}=[E]^{a} \times[f]^{b}$. Since by [So, (3.1) pg 395], $L^{\prime}$ is very ample, which happens if and only if $a>0$ and $b>a r$, and 
since $K_{F_{r}}[E]^{-2} \otimes[f]^{-2-r}$, see [Ha], we have

$$
\begin{aligned}
3 & =L^{\prime} \cdot L^{\prime}=(a E+b f) \cdot(a E+b f) \\
& =-a^{2} r+2 a b>-a^{2} r+2 a .
\end{aligned}
$$

So

$$
3>a^{2} r>r .
$$

Let $r=0$. Then $3=2 a b$, which gives a contradiction. Let $r=1$. Then $3=a(2 b-a)$. Thus $a=1, b=2$, i.e. $L^{\prime}=E \otimes f^{2}$. Therefore

$$
\hat{L}=\left(E^{-2} \otimes f^{-3}\right)^{-1} \otimes E \otimes f^{2}=E^{3} \otimes f^{5} .
$$

Let $r=2$. Then $3=2 a(b-a)$, which gives a contradiction. We can conclude that if $d \geq 9$ then $(\hat{X}, \hat{L})=\left(F_{1}, E^{3} \otimes f^{5}\right), \hat{d}=21, h^{0}(\hat{L})=18$, $d^{\prime}=3, g^{\prime}=0, \hat{c}_{1}^{2}=c_{1}^{\prime 2}=8, d \geq 9, c_{1}^{2} \geq-4, h^{0}(L) \geq 6, X$ is gotten by blowing up at most twelve points. By our previous classification we see that in the case

$$
d^{\prime}=5, \quad g^{\prime}=2, \quad \hat{c}_{1}^{2}=1, \quad \hat{d}=12,
$$

$(\hat{X}, \hat{L})$ is $\left(F_{r},[2 E+(r+3) f]\right)$, where $r=0,1,2$ with seven points blown up and, since $d \geq 9, X$ is gotten by blowing up at most three points, while, when

$$
d^{\prime}=4, \quad g^{\prime}=1, \quad \hat{c}_{1}^{2}=4, \quad \hat{d}=16,
$$

by applying Theorem (0.2) we see that $\hat{X}$ has to be $\mathbf{P}^{2}$ with at most six points blown up and $L^{\prime}=K_{\hat{X}}^{-1}$. Since $L^{\prime}=K_{\hat{X}} \otimes \hat{L}$ it follows that $\hat{L}=$ $K_{\hat{X}}{ }^{2}$. Consider the very ample line $\Theta_{\mathbf{P}^{2}}(3)$ on $\mathbf{P}^{2}$. Denote by $\hat{X}, \mathbf{P}^{2}$ with five points in general position blown up. If $\pi: \hat{X} \rightarrow \mathbf{P}^{2}$ then $L^{\prime}=\pi^{*}\left(\Theta_{\mathbf{P}^{2}}(3)\right)$ and $\hat{L}=\pi^{*}\left(\Theta_{\mathbf{P}^{2}}(-3)\right) \otimes K_{\hat{X}}{ }^{1} .(\hat{X}, \hat{L})$ gives an example of a surface satisfying our invariants. $X$ is gotten by blowing up at most seven points. We can state the following theorem.

(3.4) THEOREM. Let $X$ be a smooth, connected, projective, rational surface and $L$ a very ample line bundle on it. Suppose $\operatorname{dim} \phi_{\bar{L}}^{-}(X)=2$ and $g=5$. Then $(X, L)$ has to satisfy one of the following sets of invariants:

(1) $\hat{X}$ is $\mathbf{P}^{2}$ with ten points blown up. $\hat{L}=\pi^{*}\left(\Theta_{\mathbf{P}^{2}}(7)-10 p^{2}\right) . \hat{d}=9$, $h^{0}(\hat{L})=6, \hat{c}_{1}^{2}=-1, d^{\prime}=6, g^{\prime}=3, d=8, h^{0}(L)=5, c_{1}^{2}=-2$.

(2) $(\hat{X}, \hat{L})=\left(F_{1}, E^{3} \otimes f^{5}\right), \hat{d}=21, h^{0}(\hat{L})=18, \hat{c}_{1}^{2}=8, d^{\prime}=3, g^{\prime}=$ $0,9 \leq d \leq 21,6 \leq h^{0}(L) \leq 18,-4 \leq c_{1}^{2} \leq 8$.

(3) $(\hat{X}, \hat{L})$ is $\left(F_{r},[2 E+(r+3) f]\right)$ where $r=0,1,2$ with seven points blown up, $\hat{d}=12, h^{0}(\hat{L})=9, \hat{c}_{1}^{2}=1, d^{\prime}=5, g^{\prime}=2,9 \leq d \leq 12,6 \leq$ $h^{0}(L) \leq 9,-2 \leq c_{1}^{2} \leq 1$. 
(4) $\hat{X}$ is $\mathbf{P}^{2}$ with five points blown up. $\hat{L}=\pi^{*}\left(\Theta_{\mathbf{P}^{2}}(-3)\right) \otimes K_{\hat{X}}^{-1} . \hat{d}=16$, $h^{0}(\hat{L})=13, \hat{c}_{1}^{2}=4, d^{\prime}=4, g^{\prime}=1,9 \leq d \leq 16,6 \leq h^{0}(L) \leq 13,-3 \leq c_{1}^{2}$ $\leq 4$.

Let $g=6$.

(3.5) LEMMA. Denote by $(\hat{X}, \hat{L})$ a relative minimal pair of $(X, L)$, where $X$ is a smooth, connected, projective, rational surface such that $\operatorname{dim} \phi_{\bar{L}}^{-}(X)=2, g=6$ and $\hat{c}_{1}^{2}>0$. Then $14 \leq \hat{d} \leq 24$.

Proof. By the identity $\left(K_{\hat{X}} \cdot K_{\hat{X}}\right)(\hat{L} \cdot \hat{L}) \leq\left(K_{\hat{X}} \cdot \hat{L}\right)^{2}$ which follows from the Algebraic Index Theorem $[\mathbf{G}+\mathbf{H}]$ either $\hat{d}=7$ or $\hat{d} \geq 14$. Assume $\hat{X} \neq \mathbf{P}^{2}$. Then $\hat{c}_{1}^{2} \leq 8$. By

$$
\left(K_{\hat{X}}+\hat{L}\right) \cdot\left(K_{\hat{X}}+\hat{L}\right) \geq g+h^{2,0}-h^{1,0}-2,
$$

if follows that $\hat{d} \leq 24$. Suppose $\hat{d}=7$. Then $d=\hat{d}=7$ and $\hat{L}$ is very ample. Now applying Castelnuovo's inequality, $h^{0}(\hat{L})=5$, and by Proposition (0.3) it follows that $\hat{c}_{1}^{2}=-12$, which gives a contradiction since we are assuming $\hat{c}_{1}^{2}>0$.

(3.6) THEOREM. Let $X$ be as in the previous lemma. Then $(\hat{X}, \hat{L})$ has to satisfy one of the following sets of invariants:

(1) $d^{\prime}=4, \hat{d}=24, \hat{c}_{1}^{2}=8, g^{\prime}=0, h^{0}(\hat{L})=20$;

(2) $d^{\prime}=5, \hat{d}=20, \hat{c}_{1}^{2}=5, g^{\prime}=1, h^{0}(\hat{L})=16$;

(3) $d^{\prime}=6, \hat{d}=16, \hat{c}_{1}^{2}=2, g^{\prime}=2, h^{0}(\hat{L})=12$;

(4) $d^{\prime}=7, \hat{d}=14, \hat{c}_{1}^{2}=1, g^{\prime}=3, h^{0}(\hat{L})=10$.

Moreover, in the first case $(\hat{X}, \hat{L})=\left(\mathbf{P}_{1} \times \mathbf{P}_{1}, \theta_{\mathbf{P}^{1} \times \mathbf{P}^{1}}(3,4)\right)$. In the second case $\hat{X}$ is $\mathbf{P}^{2}$ with four points blown up. In the third case $(\hat{X}, \hat{L})$ is $\left(F_{r}\right.$, $[2 E+(r+3) f]), r=0,1,2$, with six points blown up. In the last case $\hat{X}$ is a two-sheeted branched cover of $\mathbf{P}^{2}$ with a smooth, quartic curve as branch locus with one point blown up.

Proof. By (3.5.1) and Proposition (0.1),

$$
4 \leq d^{\prime} \leq 7
$$

Let $d^{\prime}=4$. Again by Proposition (0.1),

$$
g^{\prime}=0, \quad 14 \leq \hat{d} \leq 24 \quad \text { and } \quad \hat{c}_{1}^{2}=\hat{d}-16 .
$$

Since for $\hat{d}=14,15,16, \hat{c}_{1}^{2}=-2,-1,0$, respectively, it follows that

$$
d^{\prime}=4, \quad 17 \leq \hat{d} \leq 24, \quad g^{\prime}=0 \quad \text { and } \quad 1 \leq \hat{c}_{1}^{2} \leq 8 .
$$

Because $g^{\prime}=0$, using Theorem (0.2), we have that either $\hat{X}=\mathbf{P}^{2}$ and $K_{\hat{X}} \otimes \hat{L}=\theta_{\mathbf{P}^{2}}(1)$ or $\theta_{\mathbf{P}^{2}}(2)$, or $\hat{X}=F_{r}$. Since $\hat{X}=\mathbf{P}^{2}$ cannot happen 
because $\phi_{L^{\prime}}$ embeds $\hat{X}$ in $\mathbf{P}^{5}$, the only possible cases are when $\hat{X}$ is a Hirzebruch surface. Let $L^{\prime}=E^{a} \otimes f^{b}$ with $a>0, b>a \cdot r$. Since

$$
4=(a E+b f) \cdot(a E+b f)
$$

and $b>a r$, we see that $r \geq 1$ is not possible. Now consider $F_{0}=\mathbf{P}^{1} \times \mathbf{P}^{1}$. We want a very ample line bundle $\theta_{\mathbf{P}^{1} \times \mathbf{P}^{1}}(a, b)$ such that $4=2 a b$ and $0=(a-1) \cdot(b-1)$. Thus $\hat{\theta}_{\mathbf{P}^{1} \times \mathbf{P}^{1}}(a, b)=\hat{\theta}_{\mathbf{P}^{1} \times \mathbf{P}^{\mathbf{1}}}(1,2)$. Therefore $\hat{L}=$ $\vartheta_{\mathbf{P}^{1} \times \mathbf{P}^{1}}(3,4)$. Now assume $d^{\prime}=5$. Exactly as before we have

$$
16 \leq \hat{d} \leq 20, \quad g^{\prime}=1 \text { and } 1 \leq \hat{c}_{1} \leq 5 .
$$

By Theorem (0.2), it follows that $\hat{X}$ is $\mathbf{P}^{2}$ with four points blown up. Thus $\hat{d}=20, \hat{c}_{1}^{2}=5, h^{0}(\hat{L})=16$. Hence case $(2)$ of the statement has been obtained. Now assume $d^{\prime}=6$. Exactly as before we get

$$
\hat{d}=15,16, \quad g^{\prime}=2 \text { and } \hat{c}_{1}^{2}=1,2 .
$$

Since by our previous classification $d^{\prime}-\hat{c}_{1}^{2}=4$, we have $\hat{c}_{1}^{2}=c_{1}^{\prime 2}=2$ and $(\hat{X}, \hat{L})$ is $\left(F_{r},[2 E+(r+3) f]\right), r=0,1,2$, with six points blown up. Let $d^{\prime}=7$. As before we get

$$
\hat{d}=14, \quad g^{\prime}=3, \quad \hat{c}_{1}^{2}=1 .
$$

Since $d^{\prime}-\hat{c}_{1}^{2}=6$, by our previous classification we get $(\hat{X}, \hat{L})$ is a two-sheeted branched cover of $\mathbf{P}^{2}$ with a smooth, quartic curve as branch locus with one point blown up. A possible example is given by $\hat{L}=\mathfrak{p}^{*} K_{X^{\prime}}^{-3}$ $-q^{2}$ where $\mathfrak{p}: \hat{X} \rightarrow X^{\prime}$. To complete the study of the smooth, connected, projective, rational surface, it remains to investigate the case in which $g=6$ and $\hat{c}_{1}^{2} \leq 0$.

(3.7) THEOREM. Under the above hypothesis, the minimal pair $(\hat{X}, \hat{L})$ has to satisfy one of the following sets of invariants:

(1) $\hat{d}=9, h^{0}(\hat{L})=6, \hat{c}_{1}^{2}=-2, d^{\prime}=9, g^{\prime}=5,(\hat{X}, \hat{L})=\left(F_{r},[2 E+\right.$ $(r+3) f]), r=0,1,2$, with ten points blown up. $X=\hat{X}$ and a possible example is given by $\hat{L}=\pi^{*}\left(\Theta_{\mathbf{p}^{1} \times \mathbf{p}^{1}}(7,6)-7 p^{3}-3 q^{2}\right)$.

(2) $\hat{d}=9, h^{0}(\hat{L})=5$ or $6, \hat{c}_{1}^{2}=-1, d^{\prime}=10, g^{\prime}=6, \hat{X}$ is $\mathbf{P}^{2}$ with ten points blown up. $X=\hat{X}$. A possible example is given by $\hat{L}=$ $\pi^{*}\left(\Theta_{\mathbf{P}^{2}}(13)-10 p^{4}\right)$.

(3) $\hat{d}=9, h^{0}(\hat{L})=6, \hat{c}_{1}^{2}=0, d^{\prime}=11, g^{\prime}=7, X=\hat{X}$.

(4) $\hat{d}=10, \quad h^{0}(\hat{L})=6, \quad \hat{c}_{1}^{2}=-2, \quad d^{\prime}=8, \quad g^{\prime}=4, \hat{X}$ is $\mathbf{P}^{1} \times \mathbf{P}^{1}$ with ten points blown up. $X=\hat{X}$. A possible example is given by $\hat{L}=$ $\pi^{*}\left(\vartheta_{\mathbf{P}^{1} \times \mathbf{P}^{1}}(5,5)-10 p^{2}\right)$.

(5) $\hat{d}=10, h^{0}(\hat{L})=6, \hat{c}_{1}^{2}=-1, d^{\prime}=9, g^{\prime}=5, \hat{X}$ is $\mathbf{P}^{2}$ with ten points blown up. $X=\hat{X}$. A possible example is given by $\hat{L}=\pi^{*}\left(\theta_{\mathbf{P}^{2}}(10)-10 p^{3}\right)$. 
(6) $\hat{d}=11, h^{0}(\hat{L})=7, \hat{c}_{1}^{2}=-1, d^{\prime}=8, g^{\prime}=4, \hat{X}$ is gotten by blowing up four points on the surface (2.5.2) of [So]. $X=\hat{X}$. Possible examples are $\hat{L}=\mathfrak{p}^{*} K_{X^{\prime}}^{-3}-4 q^{2}$, where $\mathfrak{p}: \hat{X} \rightarrow X^{\prime}$, or $\hat{L}=\pi^{*}\left(\Theta_{\mathbf{p}^{2}}(9)-6 p^{3}-4 q^{2}\right)$.

(7) $\hat{d}=12, h^{0}(\hat{L})=8, \hat{c}_{1}^{2}=-1, d^{\prime}=7, g^{\prime}=3,(\hat{X}, \hat{L})=\left(F_{r},[2 E+\right.$ $(r+4) f]), r=0, \ldots, 3$, with nine points blown up. $X=\hat{X}$. A possible example is given by $\hat{L}=\pi^{*}\left(\theta_{\mathbf{P}^{1} \times \mathbf{P}^{1}}(6,4)-9 p^{2}\right)$.

(8) $\hat{d}=12, h^{0}(\hat{L})=8, \hat{c}_{1}^{2}=0, d^{\prime}=8, g^{\prime}=4, \hat{X}$ is gotten by blowing up one point on the surface (2.5.2) of [So]. $X=\hat{X}$. A possible example is given by $\hat{L}=\mathfrak{p}^{*} K_{X^{\prime}}^{-4}-q^{2}$ where $\mathfrak{p}: \hat{X} \rightarrow X^{\prime}$.

(9) $\hat{d}=13, h^{0}(\hat{L})=9, \hat{c}_{1}^{2}=0, d^{\prime}=7, g^{\prime}=3, \hat{X}$ is $\mathbf{P}^{2}$ with nine points blown up. $X$ is gotten by blowing up at most one point. A possible example is given by $\hat{L}=\pi^{*}\left(\theta_{\mathbf{P}^{2}}(7)-9 p^{2}\right)$.

Proof. By the Algebraic Index Theorem as used previously, either $8 \leq \hat{d} \leq 13$ or $\hat{d} \geq 25$. By (3.4.1) $d^{\prime} \geq 4$. Suppose $\hat{d} \geq 25$. Then using Proposition (0.1) we get $d^{\prime}=4$ and $g^{\prime}=0$. Applying again Proposition (0.1) and the fact that $\hat{c}_{1}^{2} \leq 0$ we get $\hat{d} \leq 16$ which contradicts our hypothesis. Hence $8 \leq \hat{d} \leq 13$. Consider first the case $\hat{d}=8$. By Castelnuovo's inequality $h^{0}(\hat{L})=5$. Since $\hat{c}_{1}^{2} \leq 0$ implies $c_{1}^{2} \leq 0$, by the Algebraic Index Theorem $d=8$, i.e. $d=\hat{d}=8, h^{0}(L)=h^{0}(\hat{L})=5, X=\hat{X}$, $L=\hat{L}$. Thus $\hat{L}$ is very ample. Using Proposition $(0.3), c_{1}^{2}=-7$ and again by Proposition $(0.1) d^{\prime}=5, g^{\prime}=1$. Since $c_{1}^{2}=\hat{c}_{1}^{2}=c_{1}^{\prime 2}$, by our previous classification we see that this case doesn't occur. Now consider $\hat{d}=9$. Then $d=8,9$ and $h^{0}(\hat{L})=5$,6. If $h^{0}(\hat{L})=5$ then $h^{0}(L)=5$ and $h^{1}(L)$ $=0$. Thus, by [So, (2.4) pg 393], $\hat{L}$ is very ample. Thus by Proposition (0.3), $\hat{c}_{1}^{2}=-1$. Hence in this case, using Proposition (0.1) we get $d^{\prime}=10$, $g^{\prime}=6$. In the case in which $h^{0}(\hat{L})=6$, by (3.5.1) $d^{\prime} \geq 4$ and by Proposition $(0.1), d^{\prime} \leq 11$. Using again Proposition (0.1) we obtain

\begin{tabular}{|c|c|c|c|c|c|}
$\hat{d}$ & $d^{\prime}$ & $\hat{c}_{1}^{2}=d^{\prime}-11$ & $g^{\prime}=d^{\prime}-4$ & $h^{0}(\hat{L})$ & $d^{\prime}-\hat{c}_{1}^{2}$ \\
\hline 9 & 4 & -7 & 0 & 6 & 11 \\
\hline & 5 & -6 & 1 & 6 & 11 \\
\hline & 6 & -5 & 2 & 6 & 11 \\
\hline & 7 & -4 & 3 & 6 & 11 \\
\hline & 8 & -3 & 4 & 6 & 11 \\
\hline & 9 & -2 & 5 & 6 & 11 \\
\hline & 10 & -1 & 6 & 5,6 & 11 \\
\hline & 11 & 0 & 7 & 6 & 11 \\
\hline
\end{tabular}


By our previous classification we see that the only possible cases are the last three. If $d=8$, then $c_{1}^{2}=-3,-2,-1$ respectively. Moreover by Castelnuovo's inequality, $h^{0}(L)=5$. So, applying Proposition $(0.1), c_{1}^{2}=$ -7 , which gives a contradiction. Thus $d=\hat{d}=9$, i.e. $X=\hat{X}$. In the case in which $g^{\prime}=5$ as in the case $g=5$ we have obtained the possible example that we have stated. In the case in which $g^{\prime}=6$ we apply again the adjunction process. Then $d^{\prime \prime}=\left(2 K_{\hat{X}}+\hat{L}\right) \cdot\left(2 K_{\hat{X}}+\hat{L}\right)=9$ and

$$
g^{\prime \prime}=\frac{\left(3 K_{\hat{X}}+\hat{L}\right) \cdot\left(2 K_{\hat{X}}+\hat{L}\right)+2}{2}=5 .
$$

Since $c_{1}^{\prime \prime 2}=c_{1}^{\prime 2}=\hat{c}_{1}^{2}=-1$, we have that $d^{\prime \prime}-c_{1}^{\prime \prime 2}=10$. Thus, by the previous classification, we determine what $\hat{X}$ has to be and the possible examples. In the case in which $g^{\prime}=7$ we don't know anything about $\hat{X}$. Now consider the case $10 \leq \hat{d} \leq 13$. By Proposition (0.1) and (3.5.1) we get as usual the following table:

\begin{tabular}{|c|c|c|c|c|c|c|}
\hline$\hat{d}$ & $d^{\prime}$ & $\hat{c}_{1}^{2}$ & $g^{\prime}$ & $h^{0}(\hat{L})$ & $d^{\prime}-\hat{c}_{1}^{2}$ & $\hat{d}-\hat{c}_{1}^{2}$ \\
\hline 10 & 6 & -4 & 2 & 6 & 10 & 14 \\
\hline & 7 & -3 & 3 & 6 & 10 & 13 \\
\hline & 8 & -2 & 4 & 6 & 10 & 12 \\
\hline & 9 & -1 & 5 & 6 & 10 & 11 \\
\hline 11 & 10 & 0 & 6 & 6 & 10 & 10 \\
\hline & 7 & -2 & 3 & 7 & 9 & 14 \\
\hline & 8 & -1 & 4 & 7 & 9 & 13 \\
\hline & 9 & 0 & 5 & 7 & 9 & 11 \\
\hline 12 & 6 & -2 & 2 & 8 & 8 & 14 \\
\hline & 7 & -1 & 3 & 8 & 8 & 13 \\
\hline & 8 & 0 & 4 & 8 & 8 & 12 \\
\hline 13 & 6 & -1 & 2 & 9 & 7 & 14 \\
\hline & 7 & 0 & 3 & 9 & 7 & 13 \\
\hline
\end{tabular}

Now using the previous classification and the fact that if $\hat{d}=10, d^{\prime}=10$, $g^{\prime}=6$ then $K_{\hat{X}} \cdot \hat{L}=0$ and $K_{\hat{X}} \cdot K_{\hat{X}}=0$, which contradicts the Algebraic Index Theorem, we obtain the following surfaces:
(1) $\hat{d}=10, d^{\prime}=8, \hat{c}_{1}^{2}=-2, g^{\prime}=4, h^{0}(\hat{L})=6$.
(2) $\hat{d}=10, d^{\prime}=9, \hat{c}_{1}^{2}=-1, g^{\prime}=5, h^{0}(\hat{L})=6$. 
(3) $\hat{d}=11, d^{\prime}=8, \hat{c}_{1}^{2}=-1, g^{\prime}=4, h^{0}(\hat{L})=7$.

(4) $\hat{d}=12, d^{\prime}=7, \hat{c}_{1}^{2}=-1, g^{\prime}=3, h^{0}(\hat{L})=8$.

(5) $\hat{d}=12, d^{\prime}=8, \hat{c}_{1}^{2}=0, g^{\prime}=4, h^{0}(\hat{L})=8$.

(6) $\hat{d}=13, d^{\prime}=7, \hat{c}_{1}^{2}=0, g^{\prime}=3, h^{0}(\hat{L})=9$.

Exactly as before, looking at the previous classification we obtain the statement.

Now assume $\hat{X}=\mathbf{P}^{2}$. Then $\hat{L}=\mathcal{\vartheta}_{\mathbf{P}^{2}}(5)$ and $X$ is gotten by blowing up at most sixteen points. We have summarized our results in the tables on pages 6 and 7 .

\section{REFERENCES}

[A+F] A. Andreotti and T. Frankel, The Lefschetz theorem on hyperplane sections, Ann. of Math., 69 (1959), 713-717.

[Ba] H. F. Baker, Principles of Geometry, V, Cambridge University Press, 1933.

[Bo] R. Bott, On a theorem of Lefschetz, Michigan Math. J., 6, 211-216.

[Fu] T. Fujita, On the structure of polarized varieties with $\Delta$-genera zero, J. Fac. Sci. Univ. Tokyo, 22 (1975), 103-115.

$[\mathrm{G}+\mathrm{H}] \quad$ P. A. Griffiths and J. Harris, Residues and zero cycles on algebraic varieties, Ann. of Math., 108 (1978), 461-555.

[Ha] R. Hartshorne, Algebraic Geometry, Springer-Verlag, New York, 1977.

[Io] P. Ionescu, An enumeration of all smooth projective varieties of degree 5 and 6 , Preprint, Series in Mathematics Nr. 74/1981.

[N] M. Nagata, On rational surfaces I, Mem. Coll. Sci. Kyoto, (A) 32 (1960), 351-370.

[R] L. Roth, On the projective classification of surfaces, Proc. London Math. Soc., (2) 42 (1937), 142-170.

[Sa] B. Saint-Donat, On Petri's analysis of the linear system of quadrics through a canonical curve, Math. Ann., 206 (1973), 157-175.

[Sk] F. Sakai, Semi-stable curves on algebraic surfaces and logarithmic pluricanonical maps, Sonderforschungsbereich Theoritische Mathematik 40, Universität Bonn.

[S+R] J. G. Semple and L. Roth, Introduction to Algebraic Geometry, Clarendon Press, Oxford, 1949.

[So] A. J. Sommese, Hyperplane sections of projective surfaces I-The adjunction mapping, Duke Math. J., 46 No. 2 (1979).

[So $\left.\mathrm{So}_{1}\right] \quad$ On the minimality of hyperplane sections of projective threefolds, $\mathrm{J}$. Reine Angew. Math., 329 (1981), 16-41.

Received April 26, 1982.

Universita Degli Studi Dell'Aquila

VIA ROMA 33

67100 L'AQUILA, ITALY 


\section{PACIFIC JOURNAL OF MATHEMATICS \\ EDITORS}

Donald BABBITT (Managing Editor)

University of California

Los Angeles, CA 90024

Hugo Rossi

University of Utah

Salt Lake City, UT 84112

C. C. Moore and Arthur Ogus

University of California

Berkeley, CA 94720
J. DugunduI

Department of Mathematics

University of Southern California

Los Angeles, CA 90089-1113

R. FinN and H. SAMELSON

Stanford University

Stanford, CA 94305

ASSOCIATE EDITORS

R. ARENS

E. F. BECKENBACH

B. H. NeUMANN

F. WOLF

K. YosHIDA (1906-1982)

\section{SUPPORTING INSTITUTIONS}

UNIVERSITY OF ARIZONA

UNIVERSITY OF BRITISH COLUMBIA

CALIFORNIA INSTITUTE OF TECHNOLOGY

UNIVERSITY OF CALIFORNIA

MONTANA STATE UNIVERSITY

UNIVERSITY OF NEVADA, RENO

NEW MEXICO STATE UNIVERSITY

OREGON STATE UNIVERSITY
UNIVERSITY OF OREGON

UNIVERSITY OF SOUTHERN CALIFORNIA

STANFORD UNIVERSITY

UNIVERSITY OF HAWAII

UNIVERSITY OF TOKYO

UNIVERSITY OF UTAH

WASHINGTON STATE UNIVERSITY

UNIVERSITY OF WASHINGTON 


\section{Pacific Journal of Mathematics}

\section{Vol. 113, No. $1 \quad$ March, 1984}

Flavio E. A. da Silveira, Rational homotopy theory of fibrations $\ldots \ldots \ldots \ldots 1$

Donald M. Davis, Desuspensions of stunted projective spaces ............ 35

Lou van den Dries, Exponential rings, exponential polynomials and

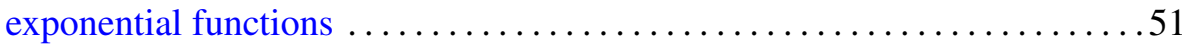

Fred Galvin and Samuel David Shore, Completeness in semimetric

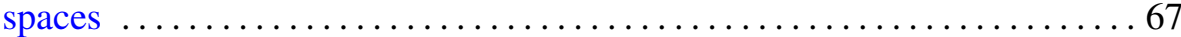

Fereidoun Ghahramani, Compact elements of weighted group algebras . . . 777

Munehiko Itōo, The closed image of a hereditary $M_{1}$-space is $M_{1} \ldots \ldots \ldots 85$

Elvira Laura Livorni, Classification of algebraic surfaces with sectional genus less than or equal to six. I. Rational surfaces .............. 93

H. Alan MacLean, Riesz sets and a theorem of Bochner ............ 115

E. Neher, Jordan triple systems with completely reducible derivation or

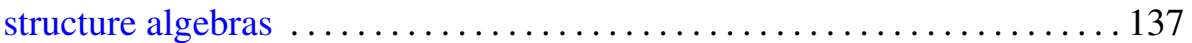

Joe Repka, Shalika's germs for $p$-adic GL( $n)$. I. The leading term $\ldots \ldots \ldots 165$

Joe Repka, Shalika's germs for $p$-adic GL( $n)$. II. The subregular term $\ldots \ldots 173$

Rae Michael Andrew Shortt, Borel density, the marginal problem and isomorphism types of analytic sets $\ldots \ldots \ldots \ldots \ldots \ldots \ldots \ldots \ldots \ldots \ldots \ldots \ldots$

Baruch Solel, The multiplicity functions of invariant subspaces for nonselfadjoint crossed products $\ldots \ldots \ldots \ldots \ldots \ldots \ldots \ldots \ldots \ldots \ldots \ldots . \ldots \ldots$

Su-win Yang, Self-intersection number of immersions and enumeration of nonstable vector bundles

W. M. Zajączkowski, Local solvability of nonstationary leakage problem for ideal incompressible fluid. II 